\title{
Study on Architecture and Implementation of Port Logistics Information Service Platform Based on Cloud Computing ${ }^{1}$
}

\author{
Changming Li, Jie Shen and Yanfang Liu \\ College of Business and Administration, Hebei Normal University of Science \\ \& Technology, Qinhuangdao Hebei Prov.066004, China \\ hbqhdlcm@126.com
}

\begin{abstract}
This paper analyses service objects and functional positioning of port logistics information service platform firstly. The architecture of port logistics information service platform upon cloud computing is proposed Based on the research of the concept and service mode of cloud computing. The system configuration and function implementation on the basis of Windows Azure Platform is further discussed in detail.
\end{abstract}

Key words: port logistics, information service platform, cloud computing, Windows Azure

\section{Introduction}

The large-scale comprehensive port which comprises inland port, airport and harbor plays an important role in leading regional economy and participating international division. Development and construction of port logistics information service platform has a positive effect to promote regional industry agglomeration and port function upgrading. Cloud computing technology can improve integration of the distributed port logistics service and realize data integration and application integration between different business systems on the port logistics data exchange platform. Therefore, this paper tries to provide useful suggestions on the construction and realization of logistics information platform based on cloud computing.

\section{Service Objects and Functional Positioning of Port Logistics Information Service Platform}

\subsection{Service Objects of Port Logistics Information Service Platform}

The service objects of port logistics information service platform mainly relates to government departments, social and financial services institutions, logistics enterprises and various cargo owners. The platform service objects are clearly illustrated in Figure 1.

\subsection{Functional Positioning of Port Logistics Information Service Platform}

Port logistics information service platform mainly provides service of logistics resource allocation, logistics resource data interaction, logistics common service support and other functions. Information sharing, resource matching, routing selection, intermodal matching, visualization of process tracking, data analysis and should be supply to the logistics related units in an efficient and integrated logistics information platform which has a friendly interactive interface can provide comprehensive logistics solutions for the

\footnotetext{
${ }^{1}$ This work was supported by the major Humanities and Social Sciences Research Project of Education Department of Hebei Province under Grant No. ZD201450.
} 
customers. [1] Main functions of the platform are as follows:

\subsubsection{The Sharing of Information Resources}

Port logistics information service platform with the function of releasing and retrieving all kinds of information related to logistics can provide consulting and checking service, which include the release of supply-demand information, Information interaction and sharing among enterprises under the supply chain etc.

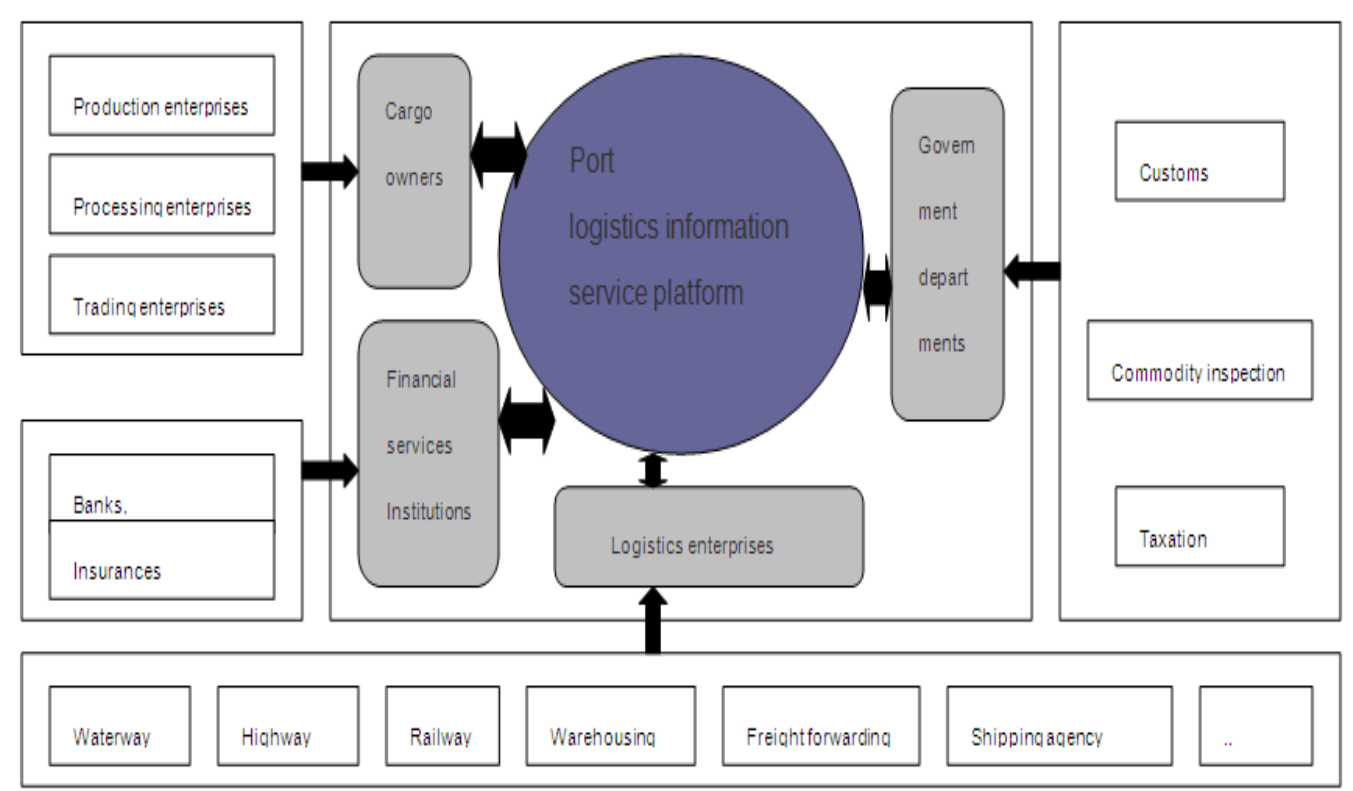

Figure 1. The Service Objects of Port Logistics Information Service Platform

\subsubsection{Electronic Trading Logistics}

The contents of electronic trading logistics comprise online negotiation, online payment, in transit tracking, cargo insurance, expense settlement ,records of trading results and statistical analysis of logistics services including all aspects such as storage, distribution, financial and others.

\subsubsection{Integrated Service}

Dynamic and standard integration mode and fruitful information resource including government, enterprises, logistics hubs and financial institution make the platform can utilize various single service functions comprehensively to provide one-stop service[2], one ticket system and a package of services such as Multimodal transport one ticket system, one-stop service of electronic trading logistics, Comprehensive supply chain solutions and so on.

The information system is heterogeneous for the participants at various levels in the logistics system and information requirements are diverse. Therefore, the distinct situations of the different participants should be fully considered in the planning and design of logistics information platform in order to accurately grasp the function that the logistics information platform should possess to ensure the availability of the platform. 


\section{Cloud Computing Services}

\subsection{Conceptual Model of Cloud Computing}

The related concept and theory of cloud computing were put forward by Google in the "Google 101 plan" for the first time in 2006. Cloud computing is the new development of distributed computing, parallel computing and grid computing. Cloud computing utilizes mainly existing resources to construct resource pool that consists of a large number of computers using virtualization technology. The resource pool is called the "cloud" which has the ability of control and supervision that can cut and allocate computational resources dynamically and delivery services to satisfy the needs of different customers. The users only need to specify the consignation to obtain necessary services without needing to understand the details of cloud services provided. The conceptual model of Cloud computing is shown in Figure 2.

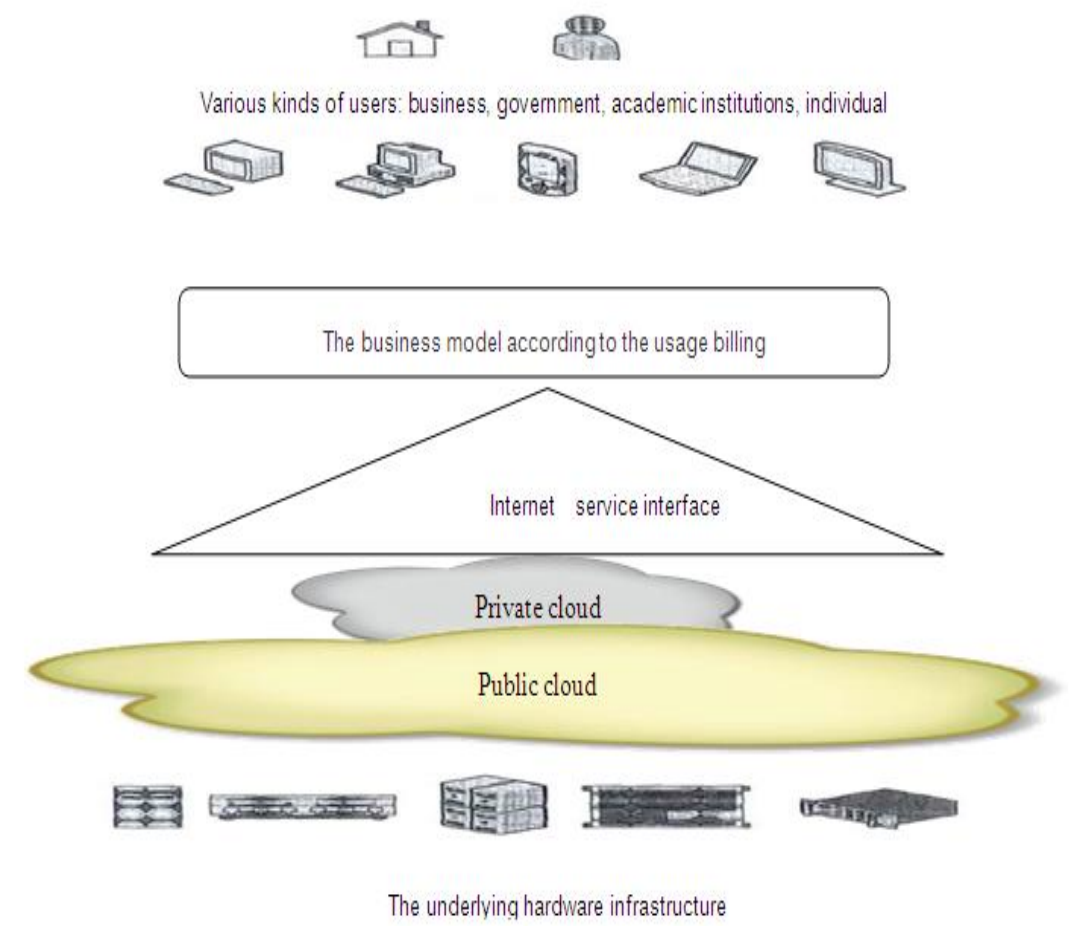

Figure 2. The Conceptual Model of Cloud computing

"Cloud" is a huge service network composed of parallel grid which extends calculation ability of the "cloud" through the virtual technology so that to maximize the effectiveness of every equipment. Processing and storage of data can be completed by the server cluster of cloud consisted of a large number of common industry standard server. A large data processing center is responsible for the management of the server cluster of cloud. Data center dynamically allocate computing resources according to the needs of customers to make the calculation spread in a large number of distributed computers, rather than the local computer or a remote server. When the users do not possess or lack of application service resource support, they can send the service request into the cloud. Then the huge data calculation and data processing tasks are placed into the cloud and the final results are return to the users. The users only need to pay the corresponding fee according to the situation of resource usage. Therefore, cloud computing is a technology of adjusting the utilization rate of resources in accordance with service mode. 


\subsection{Service Mode of Cloud Computing}

Logistics cloud computing platform consists of 3 modes: infrastructure as a service (IaaS), platform as a service (PaaS) and software as a service (SaaS) [3].

Infrastructure as a service (IaaS): Service providers will integrate storage, memory and computational capabilities into a virtual resource pool, so that they can provide services that can be measured to the customers

Platform as a service (PaaS): In this level, the cloud computing service providers furnish development environment and application server platform to the customs. The customs can develop applications of their own based on this platform and pass them to other users through the internet.

Software as a service (SaaS): Platform service providers deploy the application software on the server and the user order and rent software services according to requirement to the service providers through the network. Service providers charge such factors as time, quantity, etc. [4]

\subsection{Structure Model of Cloud Computing Services}

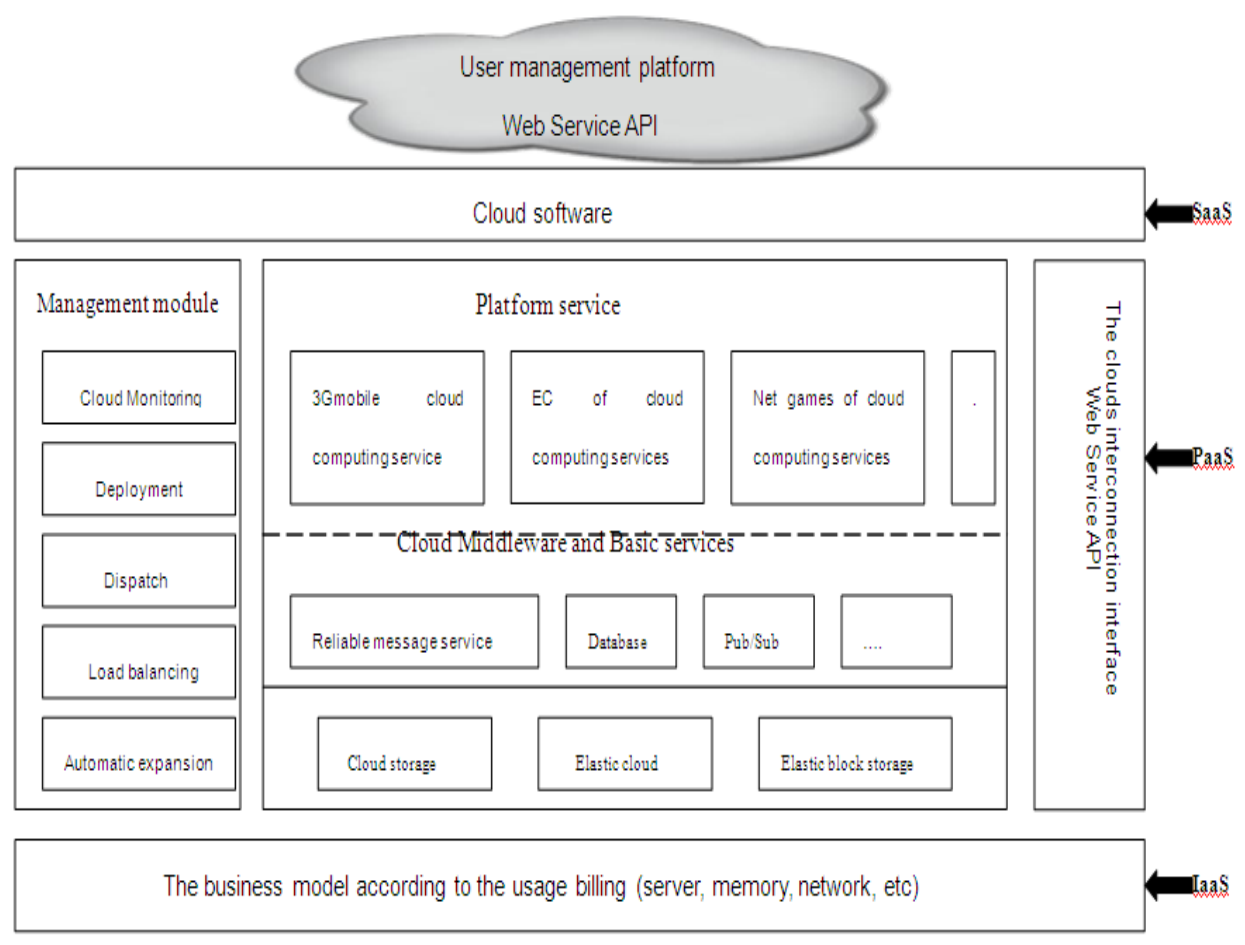

Figure 3. Structure Model of Cloud Computing Services

Structural model of Cloud computing services corresponding with service mode can be summed up through analyzing three kinds of service mode in the environment of cloud [5]. The model is shown in Figure 3. Cloud computing service Structural model is composed of underlying hardware infrastructure layer, basic management layer and application of access layer from bottom to top. Every level is described as follows:

\subsubsection{Underlying Hardware Infrastructure Layer}

The layer is the most basic components of cloud computing services in the structure model which is constructed of distinct sorts of hardware equipment from different entities. The hardware equipments interconnected via the Internet and other means of network transmission medium in the cloud computing environment are distributed in different 
geographical location according to the characteristics of cloud computing technology [6]. Each distribution hardware devices are managed by a unified management system of the storage hardware equipment in order to implement logic virtualization management, multi link redundancy management, hardware infrastructure monitoring and fault treatment and other management measures of the system [7].

\subsubsection{Basic Management Layer}

The layer is the core management component in cloud computing service structure model. Basic management layer will interconnect multiple physical devices to realize collaborative work based on Cluster system, distributed file system, Grid computing technology and other means in the cloud computing environment [8]. A unified service is provided although there may be differences in the physical device, at the same time it also can show a more powerful data access capability for its expansion.

\subsubsection{Application of Access Layer}

The layer is the component which has the highest flexibility in cloud computing service structure model. The function of application of access layer is depended on the development of the corresponding application service interface according to different business needs, thus providing different service access ability to improve the diversity and universality of the cloud computing service system. Legitimate data users authorized can access and use cloud computing service system through the exoteric Web service application interface and enjoy the convenience of cloud computing services.

\section{Architecture of Port Logistics Information Service Platform Based on Cloud Computing}

Design of port logistics information service platform architecture of cloud computing can be considered from services provided and technology. Because the existing port or logistics enterprise has its own information system, the facts of the situation should be taken into consideration combined with cloud computing, virtualization technology in the project of port logistics information service platform. The basic framework of the platform can be constructed based on the layered architecture from the bottom to the top. [9] The hierarchical architecture of port logistics information service platform is composed of physical resources layer, virtual perception layer, data resource layer, platform service layer, cloud federation service layer and application service layer from lower to upper. The specific structure is shown in Figure 4:

\subsection{Physical Resources Layer}

This layer is a cloud platform basic layer which provides the basis for virtualization of resources and services. The port can establish multiple cloud services center to build their own IaaS, also can utilize a portion of the infrastructure supplied by the third party service providers at the same time. The target of this layer is to make the platform to possess hardware foundation and have the ability of comprehensive automated information collection, the entire state monitoring and mass data processing for ships, vehicles, cargo, containers, storage and other various logistics resources.

\subsection{Virtual Perception Layer}

The layer realizes virtualization of resources and services using cloud computing and intelligent sensing technology of the Internet of things. The objective of this layer is carrying out unified organization and management for various hardware facilities including terminal equipment of Internet of things and Physical equipment of the data center and 
packaging into logic resources which has a unified interface to provide application function to ultimate user and upper layer.

\subsection{Data Resource Layer}

Effective polymerization, management and maintenance of the different types of server resources are implemented at this layer. It is convenience to schedule and manage all kinds of resources of cloud platform and improve the utilization rate of equipment and load balancing. The target of data resource layer is to carry through distributed organization, storage and management to the massive data resources through applying parallel storage management and resource scheduling technology of big data. Combined with the distributed parallel computing resource scheduling method, high performance, scalable data management model is come into being to support distributed data processing functions and provide a unified invocation interface for the upper layer application.

\subsection{Platform Service Layer}

Platform service layer offer development environment, the server platform, middleware, unified authentication, data storage, charging and other services, which is provided through a set of application programming interface(API) or facing users directly. Based on a unified programming interface provided by data resource layer, basic Web service programs can be written. The basic Web service programs are used for finishing specific function or assembling Web service system for the upper layer in order to implement management and assembly of the various Web services under the framework of SOA. The management of Web services provide publishing, finding, discovering, monitoring of the service via the service registration center mechanism. The assembly of Web services furnish data configuration interface upon business process level through a variety of methods such as working logic or business process and assemble the open and integrated logistics and related services. 


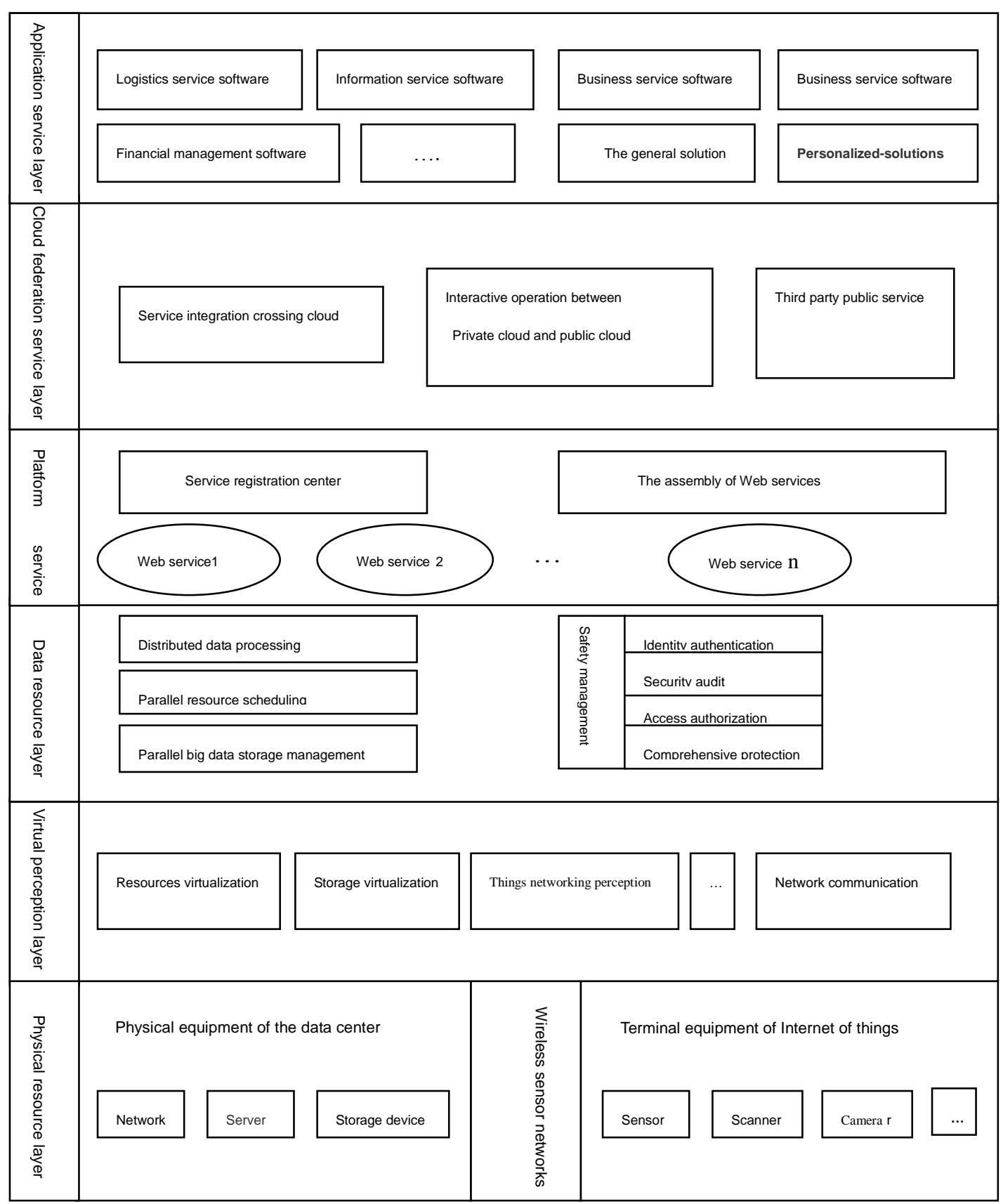

\section{Figure 4. Architecture of Port Logistics Information Service Platform Based on Cloud Computing}

Cloud Federation Services provides abstract application service by the mean of API to realize integration of cross cloud service and interactive operation between private cloud and public cloud. Local logistics system of the different port, application services upon cloud computation and third party public service are well integrated into a unified service interface supplied to the user on cloud federation service layer, so it is convenient to form a highly integrated logistics information platform to realize sharing between different ports and region. 


\subsection{Application Service Layer}

The application service layer provides the portal software service platform directly facing the terminal users, including logistics service software, information service software, business service software, government affairs service software and financial management software and so forth. In addition, port logistics information service platform can offers a variety of usage patterns through the provision of services integration interface. In order to meet the difference in demand of the clients, the layer furnish general solutions and customized solutions for customers

\section{Option and Implementation of Port Logistics Information Service Platform Based on Cloud Computing}

\subsection{Mainstream Cloud Computing Platform}

At present, there are five major platform scheme including Google cloud computing infrastructure, IBM " blue cloud" computing platform, Sun's cloud infrastructure, Microsoft's Azure cloud platform, Amazon's Elastic Compute Cloud as the mainstream cloud computing platform[10,11] .

\subsection{Option of Port Logistics Information Service Platform Based on Cloud Computing}

Because the cloud computing platform service providers are independent entities at present, each have their own cloud computing service providing mode. The access type and access method of cloud computing service system will varies according to different service providers. This paper focuses on the analysis of the Microsoft's Azure cloud platform.

\subsubsection{Components of Windows Azure Platform}

Windows Azure Platform consisted of Windows Azure, SQL Azure and Windows Azure Platform AppFabric [12].

The Windows Azure component is a development platform supporting a variety of language and environment [13]. It not only provides storage resources for developers but also provides the calculation that can be can be managed, extendible and applicative according to the requirement. At the same time, it can provide a means of control to dynamic distribute resource and data computing, storage services and operating environment for the application program deployed in the cloud [14].

SQL Azure provides relational data service to Windows Azure Platform. The data service based on the cloud hosting is a highly available and scalable.

The AppFabric component provides the interaction between applications of internal deployment and cloud applications as the distributed infrastructure services of AppFabric offering are used not only for local applications but also for cloud applications [15]. AppFabric mainly is to transmit information and connect securely between the user's free applications and cloud applications. Thus the developers focus on application logic rather than management and deployment of infrastructure.

\subsubsection{The Advantage of Windows Azure Platform}

Through the study of five cloud platform, the author suggests to choose Windows Azure Platform to develop port logistics information service platform. The advantage of Windows Azure Platform as follows:

(1) Windows Azure Platform cloud service platform running on the Microsoft data center distributed in different parts of the world provides the infrastructure for cloud 
computing and the corresponding services. [16] These services can be used solitary or combinational so that it makes the characteristics of application diversification and efficient operation. These applications can run absolutely on the cloud platform to become the cloud applications or run partially on the cloud service platform and contact with application deployed in the internal (applications that run on the data center) to improve the high availability and reliability of the application.

(2) The architecture of Windows Azure Platform has the characteristic of loose coupling which uses asynchronous message mechanism and supports a variety of mainstream standards, development language and protocol, such as.NET, Java, PHP, SOAP, REST, HTTP/HTTPS and XML etc. Three kinds of storage services (Tables, Blobs, Queues) contained by Windows Azure Platform can be accessed using standard REST application program interface. Because development mode of REST can meet the requirement of data sharing and dynamic scalability, developers can develop favorable Web application as long as they follow the PaaS architecture and get the corresponding component, function modules and SLA according to the logistics information.

(3) Platform and software tools are researched and developed independently by Microsoft, so it is self-evident in terms of compatibility. The program developers can be adept at the use of Windows Azure Platform in their professional capacity.

\subsection{Configuration of Windows Azure and Deployment of Applications}

First of all, it should be finished to configure software development environment and deploy the simulation environment of cloud application before the development of logistics management information system based on Windows Azure.

\subsubsection{Configuration of Software Development Environment}

Application development environment of Windows Azure is mainly composed of the following four parts: Visual Studio 2010, development tools supporting Azure Visual Studio, Azure SDK (Software Development Kits) and the local simulation development environment. The structure diagram of development environment is shown in Figure 5.

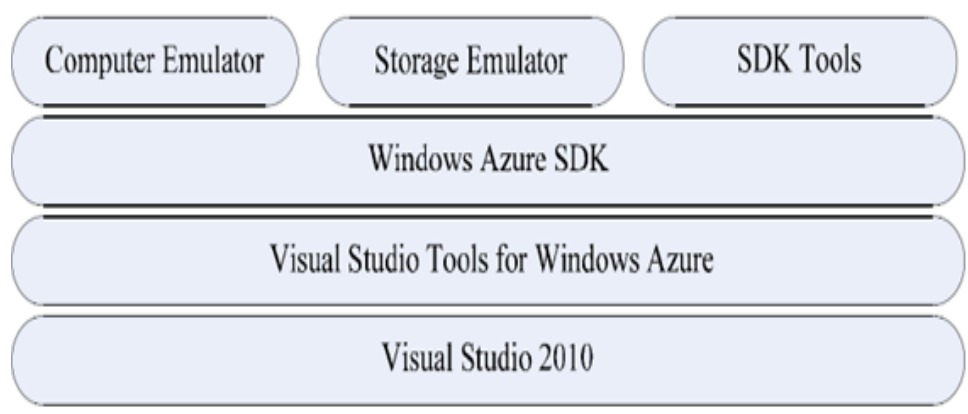

Figure 5. The Structure Diagram of Development Environment

(1) The selection of operating system: IIS service is supported to install in Windows operating system, while Fabric of Azure SDK support to operate the version above IIS7. Therefore, Windows 7(64 operation system) as the system development environment and its IIS7.0 are the choice.

(2) The selection of database: Due to varchar (max) need to be used in the Development Storage database, Microsoft Sql Server 2008 professional edition is selected in the development of the system combined with the actual development situation.

(3) The selection of development tools: Azure supporting tools are updated regularly, so Microsoft Visual Studio 2010 as the latest version of Microsoft launched is chose to use. 
(4) The selection of support tools: Visual Studio Tools for Windows Azure and Windows Azure SDK tool is the key to the development of cloud computing applications. Windows Azure SDK v1.4 can be used for this system.

\subsubsection{Deployment of Windows Azure Application Simulation Environment}

Windows Azure SDK provides a complete set of API, Compute Emulator and Storage Emulator. Developers can simulate Window Azure Fabric on local computer in order to operate and test service locally before the deployment of services.

Compute Emulator is used to simulate the cloud computing environment. The simulator will automatically run and carry out the corresponding program after the application of local Visual Studio development is opened. The user interface of Compute Emulator as shown in Figure 6.

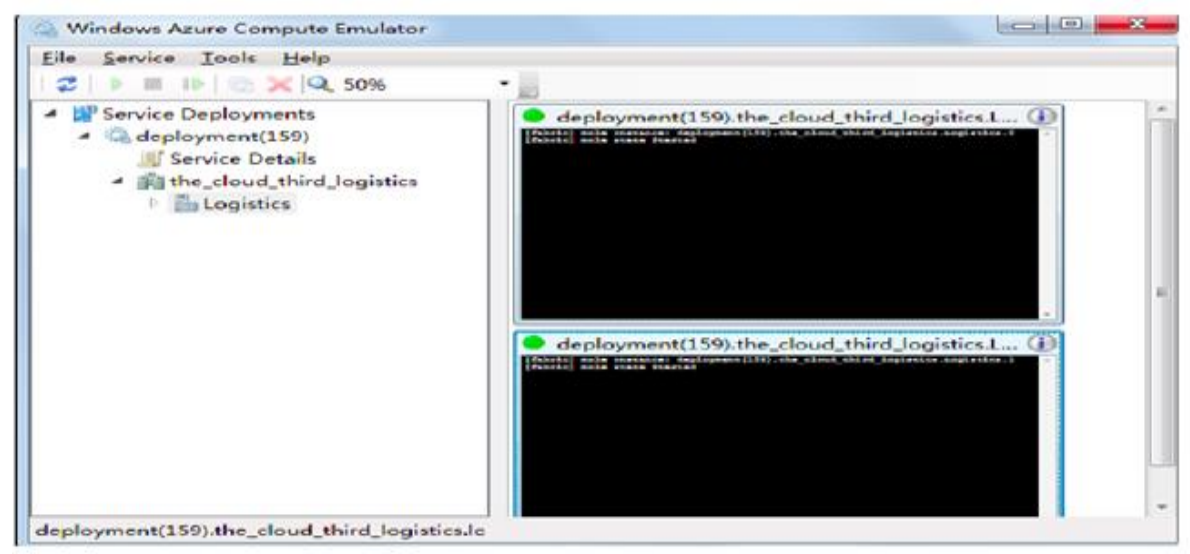

Figure 6. The User Interface of Computer Emulator

Storage Emulator is the simulator used to store local data. Azure SDK provides a REST interface for developers to access simulation database built by themselves at local.

Moreover, because the global resource locator (i.e., URLs) in the data center and the local host are not the same, the corresponding conversion environment configuration is required in the simulation of memory. Storage Emulator UI interface as shown in Figure 7.

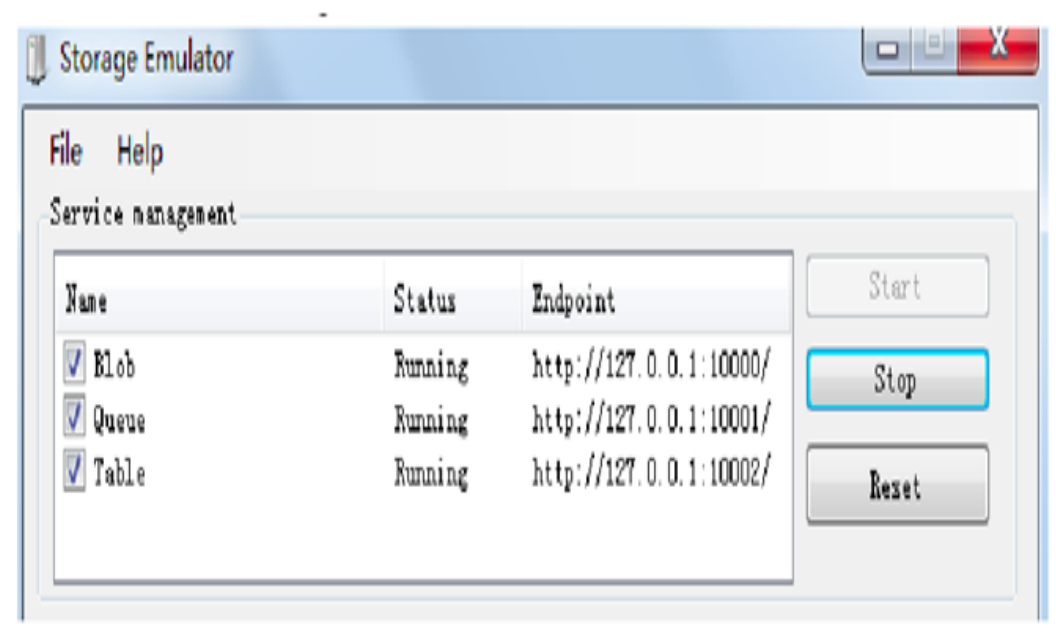

Figure 7. The User Interface of Storage Emulator 


\subsection{Implementation of Business Functions for the Platform}

\subsubsection{Development of Cloud Application}

After software development environment and the simulation environment of cloud application have been configured, developers can complete the design and development work of port logistics information service platform based on cloud computing environment in the local computing on their own computer.

The corresponding modules should be developed according to the difference of the port core function. The customers can access into the respective functional interface through the system login module, and then complete the function modules such as electronic document conversion, distribution and query of logistics information, online trading, order processing, transaction tracking and electronic payments in the corresponding function interface.

The order processing module as an example, the key procedures are as follows

In the order processing module, the users submit orders through searching the relevant goods while the administrators can review orders and finish goods delivery service timely and effectively. The key procedures of the user and administrator for processing orders are as follows:

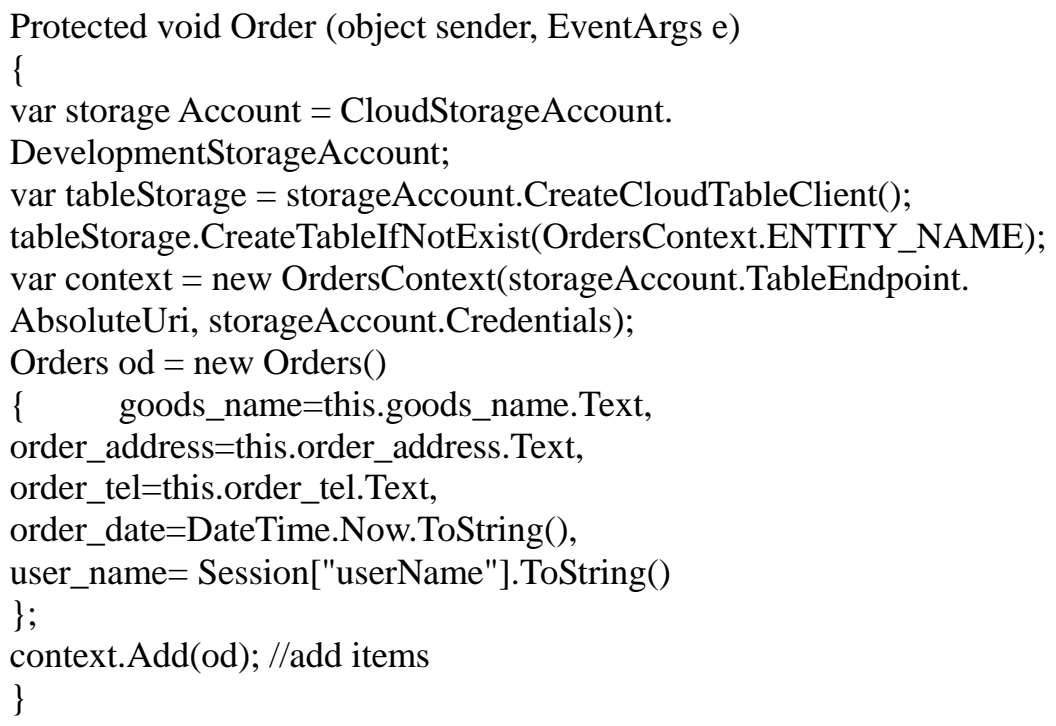

\subsubsection{The Test and Operation in Windows Azure Environment}

Testing and operating in real environments should be done after the completion of the development of Cloud Applications. The application will be put in the cloud servers to carry out the work related debugging through Windows Azure platform interface after registered Windows Live ID in Microsoft website. Relevant data storage will be done using storage service provided by Windows Azure Storage. Whether the function of the logistics information system can be realized normally is mainly assignment at this stage. At the same time testing and optimization of application performance should be done before the system put into operation formally.

$\mathrm{B} / \mathrm{S}$ architecture can be used in port logistics information service platform based on cloud computing. Integrating with the cloud resource services through the REST interface can realize the effective monitoring and management of computing resources on the cloud platform. Meanwhile certain design should be taken to ensure communication security between servers and control users access permissions effectively in order to achieve complete storage and secure transmission of data under the cloud computing environment. 


\section{Conclusion}

Modern logistics information service platform is a necessary channel to realize the informatization of port logistics and promote the development of e-commerce. This paper explores the architecture of port logistics information service platform under cloud computing service mode and strive to realize integration of resources and function of port logistics. Development and Implementation under Windows Azure cloud platform environment are researched in order to provide a useful reference for the construction of port logistics service platform

\section{References}

[1] S. Wang, "Study on Port Logistics Information Platform Based on Cloud Computation", Network and information, vol. 12, pp. 417-419, (2013).

[2] P. Zhang, "Construction of Logistics Cloud Computing Platform Based on Virtualization", Logistics Technology (Equipment), vol. 8, pp. 78-82, (2013).

[3] D. Tan, "Design and Realization of Public Logistics Information Platform under the Leadership of the Government", Transportation Enterprise Management, vol. 9, pp. 42-44, (2011).

[4] C. Jiang, "Design of cloud logistics service platform architecture for SaaS mode", Fujian computer, vol. 1, pp. 26-28, (2013).

[5] F. F. Zhang, J. T. Li and B. W. Liu, "Discussion about the Design of Logistical Public Information Platform upon Cloud Computing Framework", Business Times, vol. 30, no. 22, (2011), pp. 15-19.

[6] H. Yu, "The Construction of Logistics Information Platform Based on Cloud Computing", Science and Technology Information, vol. 27, no.1, (2010), pp. 443-444.

[7] M. Jiang, Q. Yang and C. Wu, "Network virtualization for Future Internet Architecture Design and Validation", Proceedings of 2011 3rd IEEE International Conference on Information Management and Engineering, (2011) October, pp. 556-561, Zhengzhou, China.

[8] B. Fang, "Cloud Computing Network Virtualization Technology", Information and communication technology, vol. 5, no. 1, (2011),50-53.

[9] Q. Zhao, "Research of data-exchange platform for Eurasian continental bridge logistics based on cloud computing", Technique and Method, vol. 6, pp. 79-81, (2013).

[10] J. Luo, "Cloud Computing: Architecture and Key Technologies", Journal of China Institute of Communications, vol. 32, no. 7, (2011), pp. 4-2.

[11] H. L. Truong, S. Dustdar, "Cloud computing for small research groups in computational science and engineering: current status and outlook", vol. 91, no. 1, (2011), pp. 75-91.

[12] D. Luo, "Analysis of Windows Azure Platform (Microsoft cloud computing platform), Silicon Valley, vol. 8, no. 16, (2010), pp. 9-10.

[13] H. Su, "Design and Implementation of Windows Azure Platform Based Microsoft on Cloud Computing Platform", Information Technology, vol. 1, (2013), pp. 180-181.

[14] I. Kim, J. Y. Jung, T. F. DeLuca, et al, "Cloud Computing for Comparative Genomics with Windows Azure Platform", Evolutionary Bioinformatics Online, vol. 5, no. 8, (2012), pp. 527-534.

[15] P. Nielsen and U. Parui, "Microsoft SQL server 2008 bible, vol. 6, (2011), pp. 8-25.

[16]W. Wu, "The Design of the Third-party Logistics Management System Based on Cloud Computing Platform", vol. 3, (2014), pp. 9-13.

\section{Author}

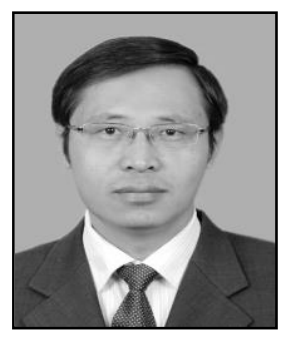

Changming $\mathbf{L i}$, received the M.S. degree from College of Management and Economics in Tianjin University. He is a lecturer who engaged in Third party logistics, Procurement management, Supply chain management and other courses teaching and research work in the College of Business and Administration in Hebei Normal University of Science \& Technology. His main research interests are research of in Logistics and supply chain management. He has published papers in the core journals and participated in scientific and teaching research projects. 